\title{
MICROSTRUCTURE EVOLUTION AND DEFORMATION FEATURE OF AZ31 MAGNESIUM ALLOY DURING SEVERE PLASTIC DEFORMATION
}

\author{
LI-FENG HOU \\ College of Materials Science and Engineering, Taiyuan University of Technology \\ Institute of Applied Mechanics, Taiyuan University of Technology \\ No.79 Yingze West Street, Taiyuan, 030024, China \\ houlifeng@tyut.edu.cn \\ YING-HUI WEI \\ College of Materials Science and Engineering, Taiyuan University of Technology \\ Taiyuan, Shanxi 030024, China \\ weiyinghui@tyut.edu.cn \\ XUE-FENG SHU \\ Institute of Applied Mechanics, Taiyuan University of Technology, Taiyuan Shanxi 030024, China \\ shuxuefeng@tyut.edu.cn
}

\begin{abstract}
A nanostructured surface layer was produced on commercially AZ31 magnesium alloy using surface mechanical attrition treatment (SMAT). The microstructure evolution and deformation feature along the depth of the treated surface layer were characterized by transmission electron microscope (TEM) investigations. The grain refinement process, accompanied by an increase in the surface layer, involves: the onset of twins; the formation of microbands associated with the dislocation slipping; the subdivision of microbands into low angle grains and then highly disoriented polygonal submicronic grains, and further breakdown into randomly oriented nanograins with progression of dynamic recrystallization.
\end{abstract}

Keywords: SMAT; magnesium alloy; nanostructure; dynamic recrystallization.

\section{Introduction}

Magnesium alloys are of the lowest density among the structural metallic materials, which makes them attractive for the automobile, aeronautical and aerospace, and electronic industries. ${ }^{1,2}$ However, magnesium alloys have poor formability and limited ductility at room temperature ascribed to their hexagonal closed-packed (hcp) crystal structure. SMAT is one of the most effective processing methods to fabricate various ultra fine-grain structures by imposing intense plastic strains onto the surface of the metals or alloys. This technique successfully achieved a gradient variation of plastic strains and strain rates in the sample from the treated top surface to the deep matrix. The microstructure observations at various depth levels can in turn provide the clue to the 
process of structural evolution relevant to various stages of strain. ${ }^{3}$ As of now, the ultra fine-grain or nano-crystalline specimens have been fabricated in several metal materials by SMAT, such as iron,4 aluminum,5 AISI304 stainless steel,6 316L stainless steel,7 cobalt, 8 titanium, 9 zirconium10 and AZ91 magnesium alloy,11 etc. The purpose of this paper is to investigate the microstructure evolution and deformation feature of AZ31 magnesium alloys by means of SMAT, and place an emphasis on the understanding of the grain refinement mechanism during the deformation.

\section{Experimental}

The alloy used in the present study was Mg-Al-Zn alloy, AZ31, with the chemical composition (in weight percent) of $3.03 \mathrm{Al}, 0.96 \mathrm{Zn}$, and $0.34 \mathrm{Mn}$. The cast ingot of the alloy was homogenized at $350^{\circ} \mathrm{C}$ for $5 \mathrm{~h}$ in the vacuum furnace. Then the ingot was cut into pieces with dimension of $100 \times 100 \times 5 \mathrm{~mm} 3$ suitable for SMAT processing. The experimental setup and the details of SMAT processing were described in Ref.3.The structural evolution of the surface layer and the deformation features of different regions in the specimens of the AZ31 were studied by H-800 transmission electron microscope (TEM). The average grain sizes were calculated using the liner intercept method.

\section{Results and discussion}

\subsection{Deformation twinning ( 200 $\mu \mathrm{m}$ below the treated surface)}

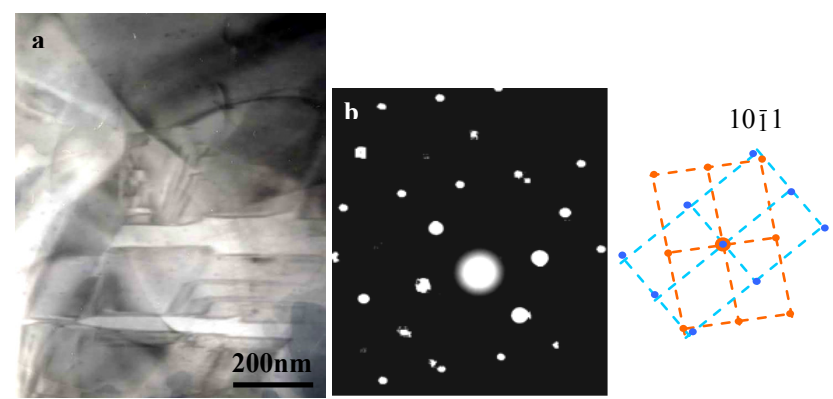

Fig.1 TEM image showing deformation twinning (a) and corresponding SAED pattern (b) with indexing.

Fig.1a is a cross-sectional bright-field TEM micrograph of the microstructure at about $200 \mu \mathrm{m}$ depth below the treated surface. The microstructure consists of parallel bands with different widths. Fig. 1b shows selected area electron diffraction (SAED) patterns taken from areas that contain adjacent bands. This reveals that they are twins, mirror spots can be observed with respect to the $\left(10 \overline{1}_{1}\right)$ plane, indicating a $\left\{10 \overline{1}_{1}\right\}$ twin system, one of the most frequent at ambient temperature. The twins generally run across the entire length of the grain and stop at the boundary, but occasionally, one of the ends terminates inside the grain. Since the relative atomic movement is limited in deformation twinning, the gross deformation produced by twins is quite small and most of the plastic flow is due to movement of the dislocations.12,13 In the experiment, the twins appear 
only in the layer adjacent to the substrate, in which the strain is low. It implies the twinning, divides the original coarse grains into finer twin platelets, governed the plastic deformation at the beginning.

\subsection{Dislocation slipping ( 150 $\mu \mathrm{m}$ below the treated surface)}

When the depth is decreased, thereby causing greater strain, a large number of very fine parallel lamellae or microbands can be observed at the depth of $150 \mu \mathrm{m}$ below the treated surface, most of which are divided into a series of blocks, exist almost everywhere, shown in Fig.2a. It illustrated that the dislocation slipping become a major strain coordinated manner to form a deformation band, which consist of several elongated subgrains. The SAED (Fig.2b) showed that the deformation band slip along the $\left[^{1^{1}} 01\right]$ direction and the misorientation of $10.2^{\circ}$ between two low angle grain boundary.
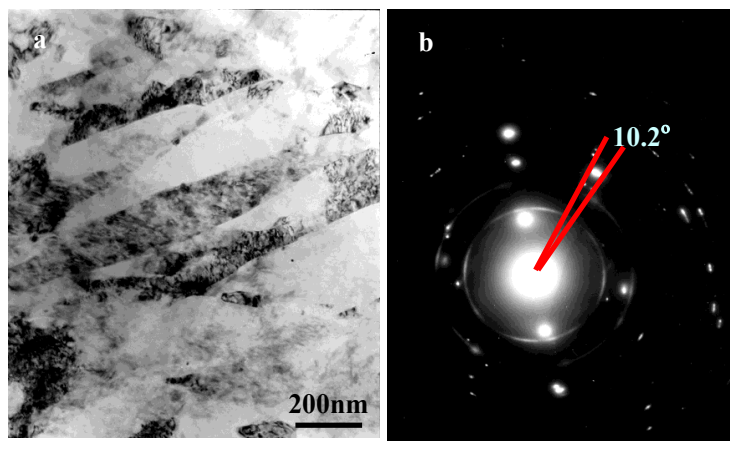

Fig.2 (a) TEM images showing the deformation microbands in the layer about $150 \mu \mathrm{m}$ below the treated surface, (b) SAED pattern from the adjacent plates labeled. $\mathrm{B} / /\left[1 \mathrm{~L}^{\overline{2}} 1^{\overline{3}}\right]$.

\subsection{Ultra-fine grains ( 100 $\mu \mathrm{m}$ below the treated surface)}

TEM examination of the layer about $100 \mu \mathrm{m}$ below the treated surface shows that the microstructure differs considerably from that observed at the depth of $150 \mu \mathrm{m}$. In some places, the fine lamellar structure has virtually disappeared and has been converted into $1 \sim 2 \mu \mathrm{m}$ submicronic grains, shown in Fig.3. There is almost no dislocations existed inside the grains. However, the residual lamellas contain a high density of dislocations, shown in Fig.4. It is observed that the dislocation walls are existed inside the microbands, correspond to a typical configuration that can accommodate plastic deformation. It is suggested that these dislocation walls are at the origin of the formation of subgrain boundaries which will subsequently be converted into low angle disoriented blocks before becoming submicronic polygonal grains. This hypothesis is supported by Fig. 4 showing the presence of submicronic grains (marked by white arrows) and the high angle grain boundaries (marked by black arrows). It is reasonable to believe that the increase in strain is the cause of the subdivision of lamellae into blocks and the formation of polygonal grains. 


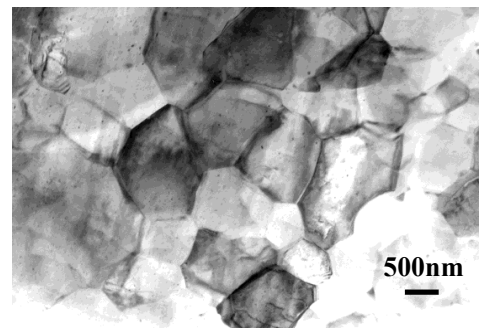

Fig.3 TEM image showing ultrafine-grains.

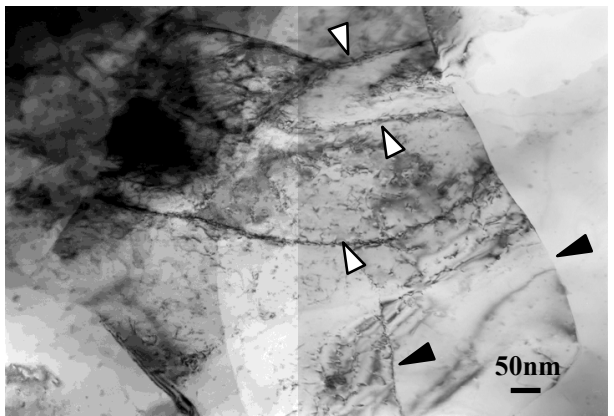

Fig.4 TEM image showing the subgrain boundary marked by white arrows and high angle grain boundary marked by black arrows deformed by dislocation interaction.

\subsection{Naocrystallization (30 10 $\mu \mathrm{m}$ below the treated surface)}

An examination of the layer about $30 \mu \mathrm{m}$ below the treated surface shows that the microstructure is the nanometer-scale grains (Fig.5). The TEM image illustrates the polygonal microstructure in the nanometer region. The average grain size is determined to be $200 \sim 300 \mathrm{~nm}$. There are a number of dislocations inside the grains. Otherwise, the clean grains existing in Fig.5a suggest that a dynamic recrystallization (DRX) process may play an important role in the formation since it occurs during the SMAT process. Derby has ever carried out14 a systematic analysis of the mechanism for DRX, and Ion has also studied15 the reason why DRX is easy to take place in magnesium alloys. In our experimental, TEM examinations do not show evidence of nucleation and growth mechanism for nanograins but indicate clearly the importance of grain boundary rotation in microstructural development. It is therefore concluded that rotation recrystallization may play a major role in the final grain refinement mechanism during SMAT. TEM examination of the top surface layer of specimen $(\sim 10 \mu \mathrm{m}$ below the treated surface) shows that the microstructure is characterized by uniformly distributed equiaxed nanometer-scale grains (Fig. 6a), the average grain size is determined to be $50 \mathrm{~nm}$. The corresponding SAED patterns of areas with a diameter of $7 \mu \mathrm{m}$ exhibit more pronounced rings than those at a depth of $30 \mu \mathrm{m}$ (Fig.5b), which illustrates the formation of finer grains with a more random orientation on the top treated surface. The diffraction rings in Fig.5b and Fig.6b have therefore been indexed and all of them are shown to correspond to the main crystallographic planes of the $\alpha-\mathrm{Mg}$ phase. 

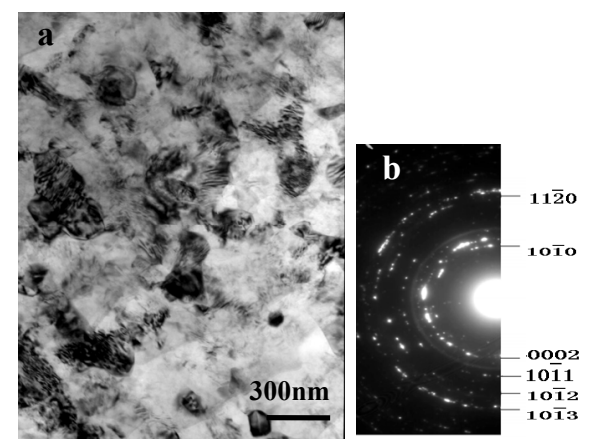

Fig.5 (a) TEM images showing submicro-crystalline (about 200 300nm) deformed by DRX;

(b) corresponding SAED pattern
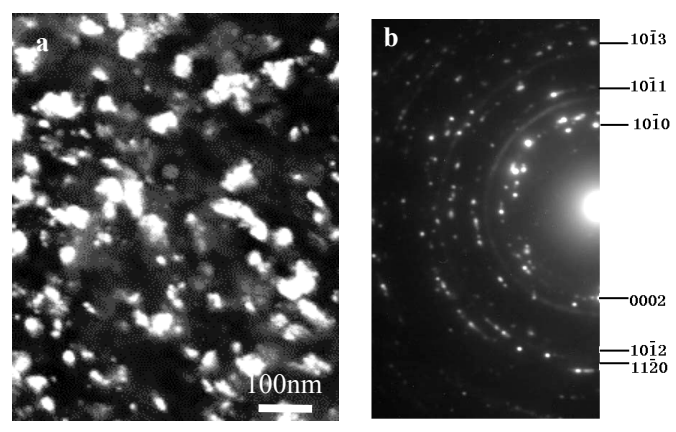

Fig. 6 TEM images of the top-surface layer (a) Dark-field image showed equiaxed nanograins; (b) Corresponding SAED pattern.

\section{Conclusions}

A Nanocrystructure can be produced on AZ31 magnesium alloy using the SMAT process. Based on the microstructural features observed at various depths and different levels of strain in the deformed surface layer, it can be concluded that, as the strain and strain rate increase, the following changes occur in the microstructure of AZ3 magnesium alloy during SMAT: (i) the onset of deformation twinning, the prevalent deformation mode at low strain in the layer adjacent to the substrate; (ii) the formation of microbands consist of elongated subgrains, associated with the dislocation slipping; (iii) the subdivision of microbands into low angle grains and then highly disoriented polygonal submicronic grains; (iv) nanocrystallization occurs along with the high strain rise and progression of DRX.

\section{Acknowledgments}

The authors gratefully acknowledge the National Natural Science Foundation of China (51001079, 51044007), the Top Young Academic Leaders of Higher Learning Institutions 
of Shanxi, the Doctoral Found of Ministry of Education of China (20091402110010), the China Postdoctoral Science Foundation (20100471586) and the National Natural Science Foundation of Shanxi Province (2011011020-2) for providing funding in support of this work.

\section{References}

1. B.L. mordike, T. Ebert, Mat. Sci. Eng.A 302,37(2001).

2. Ying-hui Wei, Bao-sheng Liu et al, J. Alloy Compd. 452,336(2008).

3. Lifeng Hou, Yinghui Wei et al, J. Mater. Sci. 43,4658(2008).

4. Tao N R, Wang Z B et al, Acta Mater. 50, 4603(2002).

5. Wu X L, Tao N R et al, Acta Mater.50,2075(2002).

6. Zhang H W, Hei Z K et al, Acta Mater. 51,1871(2003).

7. Lu Aiqiang, Liu Gang wt al, Acta Metall. Sin. 40, 943(2004).

8. Wu X., Tao N. et al, Acta Mater.53,681(2005).

9. Zhu K Y., Vassel A. Et al, Acta Meter.52,4101(2004).

10. Sun Caiyun, Wu Xiaolei et al, Transactions of Materials and Heat Treatment. 26,73(2005)

11. Lifeng Hou, Yinghui Wei et al, J Alloy Compd. 492,347(2010).

12. Reed-Hill RE, Physical metallurgy principles. (East-West Press, New Delhi, 1973) .

13. Courtney TH, Mechanical behavior of materials (McGraw-Hill, New York, 1990).

14. Derby B, Acta Metall Mater. 39,955(1991).

15. Ion S E, Humphreys F J et al, Acta Mater. 30, 1909(1982). 